for the experienced psychiatric clinician is the informed, rational use of any test that is ordered.

PETER KAMP

RICHARD B. ROSSE

Washington Veterans Administration Medical Center Psychiatry Service

50 Irving Street $N W$

Washington, DC, 20422

\section{Dyskinesia in the mentally handicapped}

SIR: It was very interesting to read the well designed study of a well defined population by Dinan \& Golden (Journal, July 1990, 157, 131-132).

We have undertaken the screening of the whole mentally handicapped population at Cell Barnes Hospital $(n=550)$ for dyskinesia. After an extensive trial period we decided to use the Dyskinesia Identification System Condensed User Scale (DISCUS) instead of the Abnormal Involuntary Movement Scale (AIMS) because of the former's superiority in identifying facial tics and grimaces, and in having separate items for the tongue movements, tongue thrust, tongue tremor and athetoid/myokymic/ lateral tongue (Sprague et al, 1989). Also, DISCUS is a standardised and validated scale for mentally handicapped people who offer little or no co-operation.

In our pilot study, the prevalence of orofacial dyskinesia in the institutionalised mentally handicapped population was $77 \%$, in accordance with Dinan \& Golden's results. Richardson et al (1986) found that their group of mentally handicapped people with tardive dyskinesia was characterised by a $92 \%$ rate of buccolingual masticatory movements.

We believe that large multicentre trials on mentally handicapped people with various psychiatric and drug histories, comparisons of various dyskinesia scores, and observations of affective changes in relation to dyskinesia should be the next step forward in the attempt to elucidate the pathophysiology of dyskinesia.

\section{Charing Cross/Westminster Medical School \\ Unit of Mental Handicap}

D. KOHEN

London W6

Cell Barnes Hospital

G. MATHEW

Highfield Lane

St Albans, Hertfordshire AL4 ORG

\section{References}

Richardson, M. A., Haugland, G., Pass, R., et al (1986). The prevalence of Tardive Dyskinesia in mentally retarded population. Psychopharmacology Bulletin, 22, 243-249.
Sprague, R. L., Kalachinik, J. E. \& Shaw, K. M. (1989) Psychometric properties of the Dyskinesia Identification System: Condensed User Scale (DISCUS). Mental Retardation, 27, 141-148.

\section{Linguistics of schizophrenia}

SIR: Thomas et al (Journal, February 1990, 156, 204 210) used methods of linguistic analysis to examine the free speech of schizophrenics and others, finding that three of the 16 descriptive variables which discriminated between clinical groups were directly concerned with 'embedding'. In an experimental study designed to investigate the psychological processes involved in the comprehension of sentences, Huff and I systematically varied 'degree of embedding' to generate 12 sentence-types with varying degrees of difficulty (Thomas \& Huff, 1971).

Each sentence specified the rooms occupied by four people (Doctor, Grocer, Lawyer, Teacher) in a house containing six numbered rooms, two on each floor. While a sentence was displayed, the subject had to put dummy 'people' into an up-ended box with six compartments so as to depict its meaning. The time taken by the subject to "do his best" (T) and the number of 'people' correctly placed $(C)$ were averaged for each sentence-type, for two groups of subjects separately and combined. The subjects were eight young adults with acute schizophrenic or schizophrenia-like illnesses and eight normal young adult controls.

High negative correlations $(-0.85$ to -0.95 , $P<0.001$ ) were consistently found between $\mathrm{C}$ and $\mathrm{T}$. This relationship was linear and essentially the same for patients and controls. The rank-order of difficulty for the sentence-types, with either $C$ or $T$ as criterion, was similar for the two groups. Evidently a single aspect of sentence structure was causing most of the difficulty, in broadly similar ways for patients and normals, although the patients performed much worse than the normals.

As the effect of 'embedding' is to make a sentence more parenthetical, we next assumed that different sentence structures imposed different amounts of 'parenthetical load' (PL) on the subject by making greater or smaller demands on the decoding processes required to extract their meaning; and secondly, that patients were more vulnerable to PL than normals. Various assumptions could be made as to the nature of the decoding processes, and each combination of assumptions (each 'model') dictated its own procedure for calculating the $\mathrm{PL}$ of any given sentence. It was consistently found that $\mathrm{C}$ correlated negatively and T positively with PL; but one model was significantly 'better' than the rest, i.e. it gave 
higher correlations, presumably because it best represented the characteristics of the decoding system.

We concluded that the comprehension of a selfembedded sentence such as "The teacher, above whom the grocer, beside whom the lawyer, above whom the doctor lives, lives, lives, lives in room six" requires a left-to-right, start-to-finish scan of the text; that each time a clause is interrupted by the beginning of another, parenthetically interpolated clause, a new temporary store is opened in memory for the new clause while the previous store remains open but quiescent; and that while material is being placed in an active store this generates interference in any previously opened, quiescent stores. In this way 'noise', arising as a consequence of the sentence structure and the way it is handled, causes the subject to make errors and also to allocate more time to the task. Individuals with intrinsically disorganised, 'noisy' minds would be especially vulnerable to this effect.

Similar mechanisms would presumably be engaged in the planning of sentences about to be uttered; so that people with 'cooler', less noisy minds should be capable of generating more complex, more parenthetical sentences; although they might not be motivated to do so and in terms of style and comprehensibility it might well be undesirable.

From this standpoint, the results of Dr Thomas et al would imply that chronic schizophrenics, although often less behaviourally disturbed than acute schizophrenics, have the more disordered, 'noisy' minds; that longer education is associated with less, and greater age with more, inherent susceptibility to parenthetical load and so on.

Parentheses are interruptions of a special kind. Dealing effectively with PL calls for the ability to place one or more uncompleted jobs 'on hold' in separate mental compartments and to insulate these as far as possible from mental activity elsewhere. This ability must be required for many kinds of thinking and in many everyday situations, as well as for handling language. A deficiency in this regard, if it is not the underlying cause of all thought disorder, must surely be an important component of it. A simplified version of our task, perhaps administered and scored by a small computer, could be of use in a variety of clinical, developmental and educational settings. It would be less time-consuming than an exhaustive linguistic analysis while still capturing much the same aspect of thought disorder, whether normal or psychotic in degree.

Middlewood Hospital

Hugh B. G. Thomas

Sheffield S6 1TP
Reference

Thомаs, H. B. G. \& HuFf, F. J. (1971) Comprehension and recording-time of transformed sentence. Language \& Speech, 14, 352-372.

\section{Understanding Capgras syndrome}

SIR: I was interested to read the report by Rastogi (Journal, June 1990, 156, 883-884) but disappointed to see once again the delusion of inanimate doubles explained away by psychological symbolism. This variant of the Capgras phenomenon is of particular significance for our understanding of delusional misidentification syndromes (Anderson, 1988).

I have seen three cases of Capgras in elderly patients during the last 12 months who all subsequently developed dementias. Global cognitive impairment had not been clinically apparent at presentation although mild impairment was demonstrated by detailed neuropsychological testing in case three.

Case one, a 76-year-old woman, developed delusional replacement of her husband three weeks after collapse, and her later cognitive decline following further collapse with right homonomous hemianopia suggested a multi-infarct pathology. Case two, an 84-year-old woman, insisted her entire home had been replaced by an identical home, and showed me each room in the house containing furniture identical to her own which belonged to the delusional owner of the house. Case three, a 79-year-old woman, refused to eat with her daughter who she maintained was an identical imposter. Cases two and three went on to develop gradually progressive cognitive decline suggestive of an Alzheimer process. Similar cases of Capgras have been described in association with dementias (Kumar, 1987; Lipkin, 1988) and most psychogeriatricians see such cases with some regularity.

An organic basis for the Capgras phenomenon is becoming increasingly likely (Cummings, 1985; Lewis, 1987; Anderson, 1988), and the continued preoccupation with psychodynamic formulations which have not furthered our understanding of these interesting conditions over the last 60 years is unhelpful and unrewarding.

\section{Fazakerly Hospital}

Longmoor Lane

Liverpool L9 7AL

D. N. ANDERSON

\section{References}

ANDERson, D. N. (1988) The delusion of inanimate doubles: implications for understanding the Capgras phenomenon. British Journal of Psychiatry, 153, 694-699. 